\title{
A COMPARISON OF LASER SCANNING AND STRUCTURE FROM MOTION AS APPLIED TO THE GREAT BARN AT HARMONDSWORTH, UK
}

\author{
D.P. Andrews*, J. Bedford, P.G. Bryan \\ English Heritage, 37 Tanner Row, York YO1 6WP, UK \\ (david.andrews, jon.bedford, paul.bryan)@english-heritage.org.uk
}

*Corresponding author

Commission V, Working Group V/2

KEY WORDS: Surveying, Laser Scanning, Photogrammetry, Structure from Motion, Medieval, Barn

\begin{abstract}
:
The great barn at Harmondsworth near London Heathrow airport, United Kingdom (UK), was built in 1426-7 for the Bishop of Winchester. At 58 metres long and 11.4 metres wide, it is one of the largest ever known to have been built in the UK, and the largest intact medieval timber-framed barn in England. The barn is built almost entirely of oak, although the walls rest on a low masonry sill-wall. Internally the space is divided into a central 'nave' with a lower aisle to each side, and is divided along its length into 12 bays. There are three doorways on the east side. For an entirely timber-framed barn, the fabric is exceptionally well preserved. Even the external weatherboarding may be partly original. Following years of neglect, however, there are a number of on-going structural and conservation problems, so in 2011 the barn was bought by English Heritage in order to allow these needs to be addressed. English Heritage is the government agency responsible for the historic sites and buildings in the care of the state of England and is also the UK government's lead advisor on the built heritage.
\end{abstract}

As one of the first steps in the conservation process the English Heritage Geospatial Imaging and Imaging \& Visualisation teams undertook a four-day campaign of survey data collection. This took the form of laser scanning of the interior and exterior of the barn plus the acquisition of photography of the exterior elevations to be used with structure from motion (SFM) software. A comparison of the results of these complimentary yet potentially competing technologies will be given, as well as an evaluation of when they can be successfully used together.

This paper will describe the procedures and problems involved with collecting the survey data and its subsequent analysis. The laser scanning was undertaken using a FARO Focus 3D phase based instrument. Approximately 60 scans were acquired in order to provide as comprehensive as possible coverage given the site circumstances. A repeat visit following the clearance of artefacts and with the benefit of access equipment was required to obtain complete coverage, especially for the top surfaces of the timber frame elements. Initial results from the laser scanning were extremely promising, with some historical events (e.g. a major fire at one end of the structure) dramatically shown in the intensity data. Comprehensive photographic coverage of the exterior of the barn including the roof was obtained using a Nikon D3X mounted on both a $6 \mathrm{~m}$ telescopic pole and a conventional tripod. A repeat visit was required to address some exposure problems in shadow areas.

A unified control network for both sets of data was obtained through the use of a total station theodolite (TST) with reflectorless electromagnetic distance measurement (REDM), incorporating a closed traverse as well as the acquisition of scanner and photogrammetric targets. The control network therefore permits the direct comparison of the results from both survey methods (allowing for systematic errors). A point cloud generated from the photography, using Agisoft Photoscan structure from motion software, was compared with the registered laser scan points with a view to determining any systematic differences, although these were to a large extent ameliorated by the use of the dense control network.

The resultant data also has potential downstream use within English Heritage for improving our understanding of Building Information Modelling (BIM) as applied to heritage structures rather than new build, and thereby contributing to the formulation of elements of a BIM strategy for English Heritage.

There are also a number of hand-measured survey drawings of the barn in existence. A quantitative as well as a qualitative comparison was made with drawings generated from the laser scan data. In general the later drawings were more metrically accurate but exhibited less understanding of the construction techniques employed. A discussion of the reasons for this is also presented. 


\section{INTRODUCTION}

Built in 1426 by Winchester College as part of its manor farm at Harmondsworth, the oak-framed barn is an outstanding example of medieval carpentry and contains one of the most intact interiors of the time. It was rescued by English Heritage, after many years of neglect, because it is Grade I listed for its exceptional architectural and historic interest. It comprises 13 massive oak cross-frames and both its size and aisles evoke the space and shape of a cathedral (Fig. 1). Indeed the famous British poet and conservationist, Sir John Betjeman described it as the 'Cathedral of Middlesex'; Middlesex being the traditional county in which it is situated (English Heritage, 2013).This epithet is particularly poignant given the barn's proximity to London Heathrow Airport, but having remained an agricultural barn for almost 600 years, the building continues to feature strongly in the life of the Harmondsworth community. Now under the management of the Friends of the Great Barn at Harmondsworth, it is regularly open to the public.

\section{LASER SCANNING}

The first step in the conservation process for the barn was the identification of the need for comprehensive recording. Drawings would be needed to help plan structural repairs and other interventions. Archaeological analysis and other academic studies would benefit and they would also be useful for recording the results of other investigations such as dendrochronology. To this end the English Heritage Geospatial Imaging Team undertook a laser scan survey while the Imaging \& Visualisation Team deployed SFM techniques.

\subsection{Fieldwork}

An initial campaign of four day's scanning with a FARO Focus $3 \mathrm{D}$ scanner resulted in as complete as possible coverage of the interior and exterior of the structure from the ground. For the interior three scans per bay, a total of 36 , were done while another 24 were required to cover the exterior. The scanner was set to acquire points at a spacing of $6.136 \mathrm{~mm}$ at a range of 10 $\mathrm{m}$ so for the interior the density was better than this in most areas. For the exterior this setting meant that the walls of the barn were captured at this spacing or better while the resolution for the, less important, roof tiles was somewhat larger especially towards the ridge.

Each scan contained a minimum of two coordinated control points in the form of checkerboard targets or hemispheres. Up to six spherical reference objects were also captured to aid registration. The control points were surveyed using a total station theodolite (TST) in reflectorless mode (REDM). Prior to the observation of the control a rigorously observed traverse around and inside the building was carried out using the TST measuring from both faces to prisms. The traverse was referenced to the British National Grid using a Global Navigation Satellite System (GNSS). However, to avoid problems with the National Grid scale factor only one survey station in the control system was fixed to the true National Grid.

A second field work session was undertaken a few months later in order to capture more of the detail of the upper surfaces of the higher timbers. To achieve this, a hydraulic lift was used to access the nave tie beams in each of the cross-frames. It was possible to mount the scanner, on a survey tri-brach secured

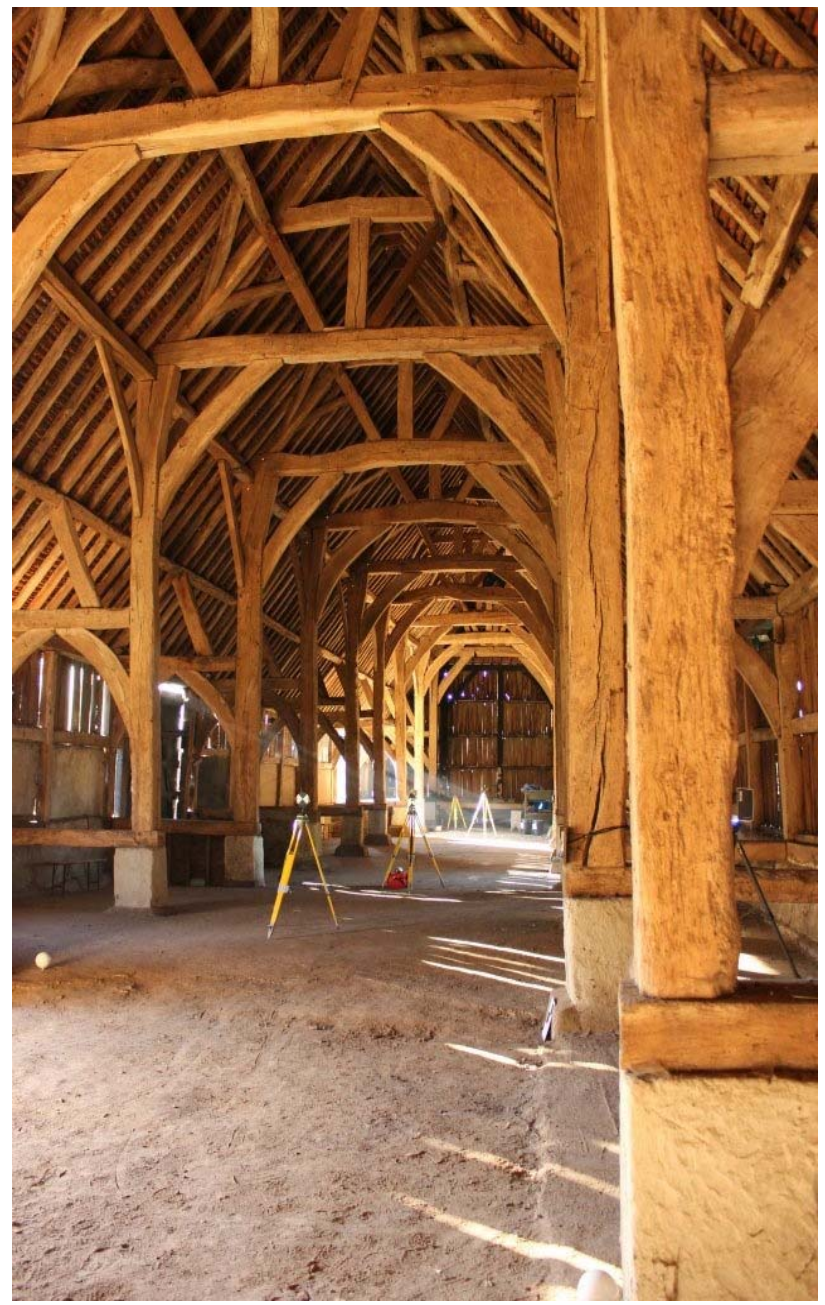

Fig. 1 The interior of the Harmondsworth Barn while the survey was in progress. Photo Bev Kerr.

with straps, on the top surface of these beams. The scanner was then controlled from the ground using the FARO remote control smartphone app. In this way another 11 scans were acquired. This time following lessons learnt in processing the first batch of scans (see below) four coordinated targets per scan were used.

\subsection{Processing}

The scans were loaded into the FARO Scene 5.1.6 software for registration and at this point a number of problems were encountered. When it came to registering the scans, acquired from the ground, it became apparent that only two coordinated points per scan is not really sufficient. This is because the procedure relies on the accuracy of the scanner's inclinometer and also there is no redundancy. The first attempt at registering all the scans together in one cluster also caused the computer to crash so the project was split into two clusters, one for the interior and one for the exterior. The two sections were registered individually using the targets and spheres as reference objects. This was a laborious job as each sphere had to be identified and correctly numbered in each scan. It was found that for the exterior scans a number of the spheres were too far away to be accurately detected by FARO Scene given that the Focus 3D scanner does not allow detailed scans of control objects within the over- 
all scan. Once all the scans were registered and the ground control file was introduced a few of the targets had quite large 'reference tensions' even though the individual fits of the scans to the control points were well within the expected tolerance, of less than $10 \mathrm{~mm}$, that would be required to meet our specification for a 1:50 scale survey. The FARO Scene manual (FARO, 2013) does not give a comprehensive definition of 'reference tensions' so it was not clear if the figures were significant. Following local advice the registration was re-done with the control file imported first. This made the process a lot easier as FARO Scene is able to automatically detect which control targets are which, using the geometry of the whole control system. This also means it can identify which non-controlled reference objects, in this case the spheres, are which. Using this method resulted in better residuals for the individual control points but some of the 'reference tensions' still seemed quite high.

\subsection{Products}

Once all the scans had been registered together it was then possible to produce the required outputs. The long-term aim of the project is to make a 3D model of the barn suitable for the application of BIM data and for use in visualisations for interpretation of, and remote access to, the barn. In the short term a number of 2D outputs have been produced to facilitate academic inquiry.

A plan (Fig. 2) and cross-section were produced in AutoCAD R 2011. The plan was achieved by exporting the scans as Bentley Pointools spod files from FARO Scene and using the Pointools plug-in for AutoCAD. Using the plug-in the scans can be loaded into AutoCAD and the apropriate view set-up. The point cloud can then be sectioned so that only the points relevant to the cut-line or other detail are visible. It is then a simple case of digitising the required line-work. The process was quite straightforward but rather slow due to the need to constantly adjust the cut-line height as the walls undulate and the floor level drops towards the southern end of the barn. The view of the point cloud is also refreshed every time AutoCAD regenerates, for example, when changing layer so this can become quite tedious. A simpler method was tried for the cross-section whereby a view was set-up in Pointools Edit Pro1.5 and the image exported as an ortho-image. The ortho-image was then attached to an AutoCAD drawing and the line work digitised. This was successful and rapid but would only really work for sections and elevations where only one view is required. Another software that works with AutoCAD, Virtusurv 5.0.0.8 from Kubit, was also trialled for the cross-section with mixed results. With this program the scans are viewed in as an un-wrapped panorama from the scan position. Line work can be digitised directly in 3D by snapping to the scan points and the coordinates are sent to AutoCAD to build up the drawing. Whilst the panorama view makes it easy to see the detail that requires digitising actually achieving the desired lines is more difficult. This is because unless a very fine spacing is used a scan point is often missing from the edge to be drawn. As a result the points digitised along the edge of a beam, for example, were often misplaced.

In order to visualise the entire long-section of the barn without the need to digitise every beam and rafter an ortho-image was again produced using Pointools Edit. This time the intensity of the laser reflection was represented by a colour ramp. The colours were brightest where the intensity was lowest and so highlighted the southern area of the barn where the timbers are blackened due to a fire in the 1970s.

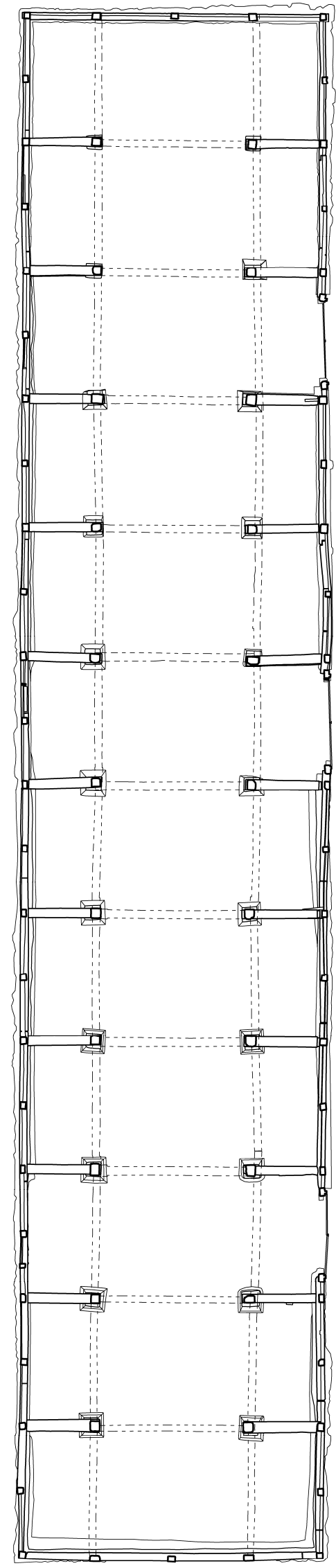

Fig. 2 Plan of the barn produced using Pointools plug-in for AutoCAD 


\section{STRUCTURE FROM MOTION}

\subsection{Fieldwork}

The photogrammetric fieldwork was undertaken at the same time as the laser scanning, although only two days were spent on data acquisition, delays largely being caused by variations in ambient lighting conditions. Photography was acquired using a Nikon D3X digital SLR. A total of 934 images were acquired for the full dataset, comprising both Nikon .raw and medium resolution .jpg files for each exposure. Three lenses were used: a Nikon Nikkor AF $28 \mathrm{~mm} \mathrm{f} / 2.8 \mathrm{D}$, with the focus set to infinity and taped down, a Nikon Nikkor AF-S Zoom 14-24mm f/2.8G ED AF taped at $18 \mathrm{~mm}$, with the focus set to infinity (both used in entirely manual modes on the camera, and with no focussing adjustment) and lastly a Nikon Nikkor 28-300mm f/3.5-5.6 VR with no restrictions on focal length or zoom was used for fill-in photography. This provided consistency of calibration for most of the shots, with the software able to estimate distortion parameters for the unrestricted photography from EXIF data.

Control was provided by temporary circular targets, with a diameter of approximately $1 \mathrm{~cm}$, applied to the barn exterior. The control points were surveyed using a Leica TS15i 3" instrument, with an accuracy of approximately $+/$-3mm given considerations of range, angle and target quality. Images were taken such that at least four control points were visible in each image. A total of 80 control points were surveyed over the entire exterior of the barn. For photographs taken from the ground the camera was mounted on a standard photographic tripod, and the photographs taken as a series of overlapping stereo pairs with approximately $60 \%$ overlap between each image and its companions on either side (Fig. 3). Separate runs were performed to include the wall faces and the shots necessary to get under the eaves of the roof to allow the rafters to be seen in the model. A further series of photographs was taken using a $6 \mathrm{~m}$ telescopic mast with the camera mounted on top. The shutter was released from the ground via a Hahnel Inspire LiveView remote control, which also showed the camera's field of view. Given the weight of the camera, the $28 \mathrm{~mm}$ lens (i.e. the lightest) was used for the mast photography. The mast allowed for coverage of the exterior of the roof as a series of photographs approximately perpendicular to its surface. The photographs were taken in runs, and attempts were made to keep ambient lighting conditions consistent for each run. Problems will be encountered in low light conditions with the camera on a mast, and for this reason interior photography was either lit artificially for consistency, or the unrestricted lens used with the camera set to shutter priority, allowing a sufficiently fast shutter speed to be used to avoid blurring, although this is sometimes necessarily at the expense of noise in the resultant images when using ISO values of 400 or higher. Using wider apertures is also a potential help, although they inevitably lead to a reduced depth of field, so the camera was stopped down as far as was reasonably practicable.

\subsection{Processing}

The photographs were processed using Agisoft's Photoscan Professional 0.9.0. Raw files were first converted to an uncompressed .tif format using Adobe Camera Raw. The photographs were first masked before the alignment process to remove potentially spurious data for the matching algorithms, i.e. the sky and elements, such as vegetation, that may have moved between exposures. The data were divided into chunks, covering the roof surface, the upright walls, the eaves and the two ends of the barn.

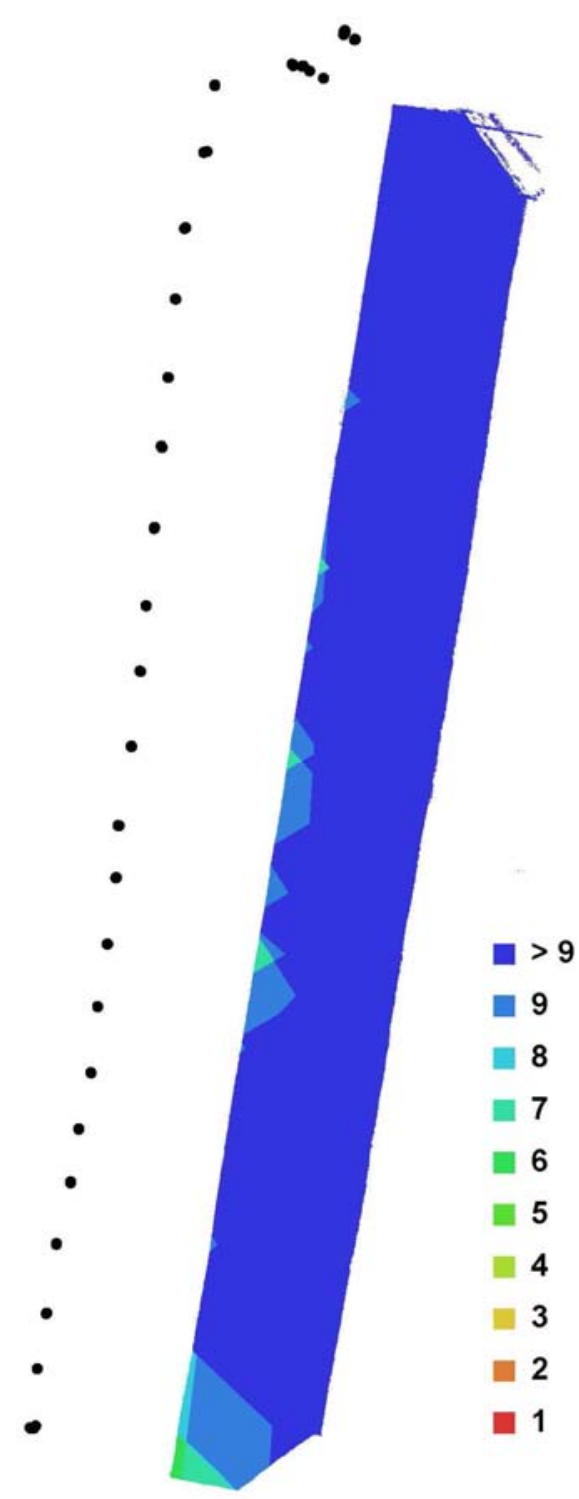

Fig. 3 Camera locations and image overlap for the roof. Lower values towards the southern end are ameliorated by additional images from adjacent chunks in the overall model.

The photographs were then aligned without using control points. This generated a series of sparse point clouds, which were iteratively filtered for reprojection errors (residuals) and optimised until estimated reprojection errors were approximately $0.5 p x$ per chunk. The masks were then added to, removing those parts of the images that had been potentially useful for the alignment process but which were not required for the generation of geometry. Low resolution geometry was then created, enabling the semi-automated placement of markers in the scene. Once marker placement was complete, the variance between their measured and estimated positions was between $0.1 \mathrm{~mm}$ and $3 \mathrm{~mm}$, or approximately $0.3 \mathrm{px}$, which is commensurate with the accuracy with which they could be measured in the field. The point cloud was again optimised. At this point the high resolution geometry was generated for each chunk, using appropriate settings and with no upper limit on the polygon count specified. This produced a fairly large file (c. 23GB for the five chunks covering the half of the barn being compared at full resolution). 


\subsection{Products}

In similar fashion to the scan data, orthophotographs of the barn exterior were generated. Given the nature of the inputs, with an average pixel ground size of approximately $2 \mathrm{~mm}$ across all chunks, these were of sufficiently high resolution, and certainly in excess of the requirements of the purposes of generating paper based outputs. The orthophotographs allowed the digitising of elevation data from them. As well as this, both the point cloud and the model generated could be used in exactly the same way as a point cloud generated by any other means, and thereby be used to generate plan and sectional data. The photogrammetric element of the survey was primarily intended as a test for the deployment of this technique as an alternative and complementary potential production drawing and modelling workflow for English Heritage. Software that is usable by nonphotogrammetric experts, with appropriate guidance, that has an understandable and relatively simple user interface, and which can produce consistent results was desirable. Another important factor concerns processing the data locally, rather than cloud processing. There are several cloud-based processing solutions currently available, but the potential longterm storage of the data outside the EU makes some of these facilities problematic or impossible to use, as English Heritage is currently an agency of the UK Government Department of Culture, Media and Sport and is therefore subject to current EU data protection laws.

\section{COMPARISON}

\subsection{Coverage}

There were clear differences in the coverage offered by the two techniques, and the relative ease with which they could be achieved. For example, coverage of the exterior of the roof from the scan positions on the ground was relatively poor, whereas it was simple to raise the camera to a point where the coverage was excellent. This effect was most notable for the laser scanner in areas where the stand-off from the building was heavily restricted by other encroaching structures. Post spacing for most of the roof, therefore, was below the interior average of $6 \mathrm{~mm}$ at $10 \mathrm{~m}$ due to the stand-off necessary to capture any of the roof at all. The time taken to acquire the data was significantly less for the photogrammetric approach, although it should be noted that this applies to the barn exterior only apart from a few small details, test data for the interior was not acquired, and would clearly represent a more complex task than the exterior.

Given the resources to hand, the data from both sources had to be decimated in order for a comparison to be made as the scan data comprised a 34GB file covering the entirety of the exterior only, with the photogrammetric data weighing in at 23GB. Both sets of data were therefore decimated to approximately 40 million polygons for the comparison. It should be emphasised that the focus was on comparing the practical application of the techniques and their relative performance at scales of output that were meaningful for archaeological research and analysis rather than the purely theoretical. It is acknowledged that there are differences in the algorithms and methods used by different software packages to generate mesh geometry, as well as estimates of noise in the dense point clouds from different sources (clearly the sensor for the photogrammetric work is broadly speaking much more variable that that of the scanner, which makes estimations on much more than a case-by-case basis very difficult) and that these will also play some part when comparing the data. A direct comparison of the dense point cloud products would also be possible using an open source product e.g. Cloud Compare (Girardeau-Montaut, 2013).

\subsection{Results}

The two meshes were compared using Geomagic Qualify 2013. Since both sets of data had been referenced to the same coordinate frame direct comparison was possible without the geometric translation of either set.

Results were extremely encouraging. Variation between the data sets was not large, and although some processing remains to be done to integrate the photogrammetrically derived data into a complete model, this will certainly be a worthwhile exercise, and will contribute significantly to the completeness of the overall model. Variance between the two datasets where they overlapped averaged $0.0197 \mathrm{~m}$ (positive $0.0337 \mathrm{~m}$, negative $0.0448 \mathrm{~m}$ ). Standard deviation for the comparison was $0.0376 \mathrm{~m}$, with an RMS estimate of $0.0425 \mathrm{~m}$, skewed in part by a maximum deviation between the data sets of $0.445 \mathrm{~m}$ accounted for in part by the necessarily short stand-off for the scanner at certain points around the structure and difficulties encountered meshing data under the eaves of the roof. The average of c. $2 \mathrm{~mm}$ variation in overlap is well within the tolerance that was considered sufficient given the factors already discussed, e.g. the tolerances of measurement on the control network and the accuracy of output required.

\section{CONCLUSIONS}

\subsection{Data capture}

The photogrammetric data was faster to acquire, and appears to have generated outputs that are of a similar order to those generated by the scanner. Raw data producing outputs of a consistently high resolution could be captured with relative ease provided one is mindful of the quality of the inputs (since the output is derived from them and therefore directly related to them) and checks regularly for consistency and completeness of coverage as well as blunders. This is particularly important with the mast photography. Furthermore, as has been demonstrated many times, the sensor and software required is far cheaper. The technique proved successful for the exterior of the barn, and in particular the roof exterior, and has generated results which we can confidently use. It is therefore possible to selectively combine the data from both sources into a consistent whole with a more even spread of data points across the entire model.

However, having processed, at least in part, some elements of the interior using the photogrammetric inputs, it is estimated that this would take considerably longer than the exterior, largely due to the complex nature of the structure and the large number of occlusions. This may to some extent be ameliorated by the use of a high-quality $360 \times 180$ degree imaging device such as the Spheron VR, the outputs of which can be processed by PhotoScan, thereby getting around the problem of having to take such large quantities of individual images and getting the time required for the photographic workflow for complex internal structures to be much more similar to that required for laser scanning, with the additional benefit of good performance in low light conditions, such as those experienced at Harmondsworth. The best overall coverage has been obtained when using 


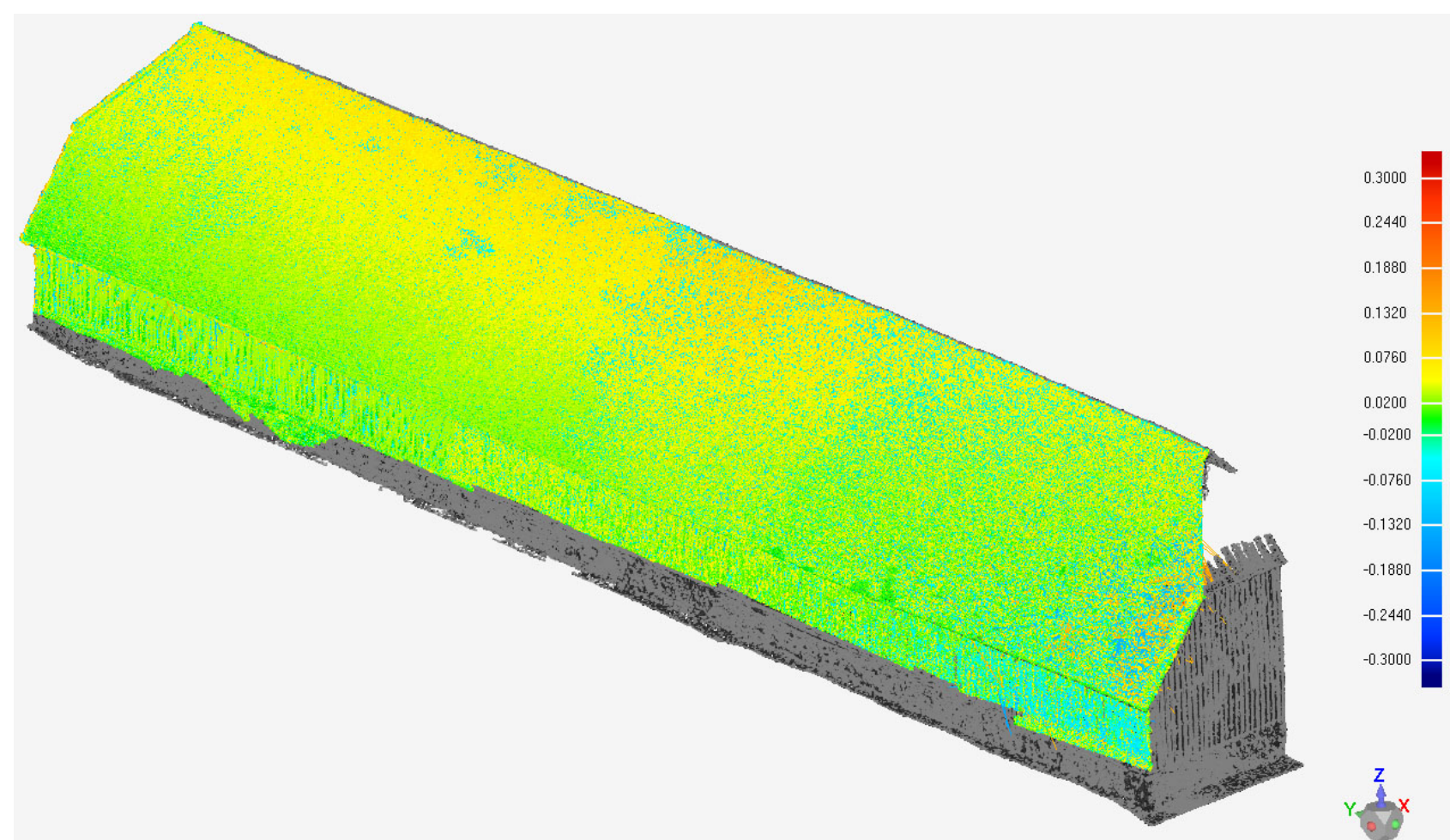

Fig. 4 Differences between the laser scan and SFM point clouds. The difference tends to increase towards the ridge due to the laser scans only being obtained from the ground while a mast was used for the photography.

the techniques in a complementary fashion, combining the results of the two, and using one to fill in where the other is weaker and vice versa. The use of the same coordinate frame for both methods allows this to take considerably less time than a best fit estimation of the point clouds or meshes. Photography of details taken inside the barn to record certain features (e.g. graffiti, mason's marks and tooling marks) produced excellent results and could be incorporated into the scan data.

\subsection{Building Information Modelling}

The early intention of this work was to use the barn as a test case for the application of BIM. The varied and undulating nature of the timbers will undoubtedly be a challenge to model; especially if the data file is not to become so big as to be unusable. However, the English Heritage BIM Special Interest Group has decided to focus on buildings that will present a greater challenge with regard to the other aspects of BIM as applied to historic buildings, for example, the identification of materials and construction techniques that are obscured by decorative finishes. Another aspect of BIM that will not be thoroughly addressed by modelling the barn is the location and depiction of services; the only service in the barn is the electricity supply and all the cables, fittings etc are temporarily fixed to the surfaces of the timber frame.

\subsection{Previous Survey}

Production of the plan and cross-section allowed a comparison with existing surveys to be made. The existing plan and section had been hand-measured and drawn in a squared-up schematic fashion. As a result the straight lines do not reveal the structural problems caused by the subsidence of the east sill-wall. This is causing the posts to rotate outwards leading to failure in the cross-frame joints. The bulge in the east wall can clearly be seen in the new plan and the failure of the joints is shown in the cross-section drawing. The intention of the hand measured drawings may have been to show an idealised view and the failure modes are indeed described in the accompanying report along with analysis of the joints etc but an accurate plan would have highlighted the structural problems.

\section{REFERENCES}

English Heritage, 2013. Harmondsworth Barn http://www.englishheritage.org.uk/daysout/properties/harmondsworth-barn/ (accessed $2^{\text {nd }}$ May 2013)

FARO, 2013. Scene Version 5.1 User Manual. FARO Technologies Inc, Lake Mary FL, section 5.6

Girardeau-Montaut, D.,2013. CloudCompare

http://www.danielgm.net/cc/ (accessed $2^{\text {nd }}$ May 2013).

\section{BIBLIOGRAPHY}

AGISOFT, 2012. Agisoft PhotoScan User Manual: Professional Edition, Version 0.9.0. Agisoft LLC, St Petersburg

Baker, TFT., et al, 1971. A History of the County of Middlesex: Volume 4: Harmondsworth, Hayes, Norwood with Southall, Hillingdon with Uxbridge, Ickenham, Northolt, Perivale, Ruislip, Edgware, Harrow with Pinner. Victoria County History, London 